\title{
Universal Quantum Cloning in Cavity QED
}

\author{
P. Milman ${ }^{1 *}$, H. Ollivier ${ }^{2,3 \dagger}$, and J. M. Raimond ${ }^{1}$ \\ ${ }^{1}$ Laboratoire Kastler Brossel, Département de Physique de l'Ecole Normale Supérieure, \\ 24 rue Lhomond, F-75231 Paris Cedex 05 France \\ ${ }^{2}$ LANL, T-6 MS B288, Los Alamos NM 87544, USA. \\ 3 INRIA-Projet CODES, B.P. 105, F-78153 Le Chesnay Cedex, France. \\ ${ }^{4}$ Collège de France, 11 place Marcelin-Berthelot, F-75005, Paris, France
}

\begin{abstract}
We propose an implementation of an universal quantum cloning machine [UQCM, Hillery and Buzek, Phys. Rev. A 56, 3446 (1997)] in a Cavity Quantum Electrodynamics (CQED) experiment. This UQCM acts on the electronic states of atoms that interact with the electromagnetic field of a high $Q$ cavity. We discuss here the specific case of the $1 \rightarrow 2$ cloning process using either a one- or a two-cavity configuration.

03.67.-a 03.65.-w 42.50.-p
\end{abstract}

a. Introduction. Quantum theory provides new and unexpected effects when compared to classical physics. Among them, the no-cloning theorem, derived in 1982 by Wooters and Zurek [1] , plays a particularly important role: While classical information can be copied perfectly and many times, quantum information cannot. This fundamental difference is a consequence of the unavoidable creation of quantum correlations. Since perfect cloning is not possible, an important question naturally arises : What is the best quantum copying operation? The answer to this question is context-dependent. On the one hand, there is a single transformation that produces the best identical copies of a qubit prepared in any input states. This "universal quantum cloning machine" (UQCM) has been discussed for the first time in [2]. On the other hand, many other rules of the game can be considered, such as state dependent cloning [3], cloning of 3-dimensional states [4] and cloning of orthogonal qubits [5].

The quality of a copy is usually measured by the quantum fidelity [6]. This quantity is discussed, in the context of universal quantum cloning machine (UQCM), in [2] and [7]. When $M$ copies are produced from $N$ identical pure 2-dimensional states, the fidelity of the copies is given by $F(N, M)=(N M+N+M) /(M(N+2))$. For the simplest case of two copies produced from one input state, this expression reduces to $F(1,2)=5 / 6$. The complete understanding of the fidelity behavior versus $N$ and $M$ is still a subject of debate, with connections to the measurement and state estimation problems [8]. Beyond these fundamental problems, the interest of quantum cloning machines also encompasses a wide area of quantum information processing, including quantum cryptography, teleportation [9], eavesdropping, state preservation and measurement-related problems, as well as quantum algorithm improvements 10].

*Electronic address: Perola.Milman@lkb.ens.fr

${ }^{\dagger}$ Electronic address: harold.ollivier@polytechnique.org
The derivation of the optimal UQCM transformation led to several proposals [11 for its experimental implementation. Most of them, based on the Buzek and Hillery quantum logics network 12, use the quantum optics framework. Experimental quantum cloning has been realized up to now only with photons as the carriers of quantum information. This information was either encoded in different degrees of freedom of the same photon (polarization and position) [13] or in the photon polarization only [14]. An alternative network adapted to NMRbased quantum information processors has also been proposed and experimentally implemented [15].

In this paper, we propose an implementation of the $1 \rightarrow 2$ UQCM operating for atomic states in the Cavity QED (CQED) context [16]. The quantum information is coded on electronic levels of long-lived highly excited Rubidium (Rb) atoms. Our protocol realizes, with four atoms, the transformation described in [2], with an original quantum logics network based on the resonant interaction between the atoms and two high- $Q$ niobium superconducting microwave cavities $C_{a}$ and $C_{b}$. We discuss, at the end of this paper, an adaptation of the scheme using two different modes of a single cavity 17, making the proposal implementation more realistic with the present cavity QED set-up. This paper focuses on the quantum logics protocol. The interested reader can find more details about the experimental techniques in [16]

Let us first recall the optimal $1 \rightarrow 2$ UQCM transformation [2]. When the qubits are encoded in the basis $\{|+\rangle,|-\rangle\}$, the UQCM performs the transformations

$$
\begin{aligned}
& |-\rangle|\mathcal{B}\rangle \rightarrow \sqrt{\frac{2}{3}}|-\rangle|-\rangle|\mathcal{A}\rangle+\sqrt{\frac{1}{3}}|\Phi\rangle\left|\mathcal{A}_{\perp}\right\rangle \\
& |+\rangle|\mathcal{B}\rangle \rightarrow \sqrt{\frac{2}{3}}|+\rangle|+\rangle\left|\mathcal{A}_{\perp}\right\rangle+\sqrt{\frac{1}{3}}|\Phi\rangle|\mathcal{A}\rangle,
\end{aligned}
$$


where the first ket of the l.h.s represents the input qubit and $|\mathcal{B}\rangle$ is the initial state of the blank copies and of possible ancilla qubits involved in the process. In the r.h.s, the first two kets are the quantum clones, $|\Phi\rangle=$ $(|+\rangle|-\rangle+|-\rangle|+\rangle) / \sqrt{2}$. The third ket represents two possible orthogonal final states, $|\mathcal{A}\rangle$ and $\left|\mathcal{A}_{\perp}\right\rangle$ for the ancilla qubits.

Our scheme makes use of three atomic levels, $|e\rangle,|g\rangle$ and $|i\rangle$. The transition between levels $|e\rangle$ and $|g\rangle$ can be set in and out of resonance with the cavity modes, using the Stark effect induced by an electric field applied between the Fabry Perot cavity mirrors [16]. The auxiliary level $|i\rangle$ is far off-resonant from the cavity fields and is not coupled to them. However, it can be accessed via classical microwave pulses either from level $|g\rangle$ (one photon transition) or from level $|e\rangle$ (two-photon transition). The atomic qubit encoding is $|+\rangle=1 / \sqrt{2}(|i\rangle+|g\rangle)$ and $|-\rangle=1 / \sqrt{2}(|i\rangle-|g\rangle)$. The photon number states of each cavity mode is denoted as $|n\rangle_{i}$, where $i=(a, b)$.

$b$. Description of the protocol: The sequence of operation achieving the UCQM transformation is pictorially depicted in Fig. (11). It presents, in a space-time diagram, the space lines of the two cavity modes and of the four Rydberg atoms, $A_{1-4}$, involved in the process. The atom-cavity resonant interactions are represented by black lozenges. Classical microwave pulses mixing the atomic levels are represented as gray circles.

The cavity fields are initially prepared in the vacuum state $|0\rangle_{i}$ 16]. The first atom, $A_{1}$, intially in state $|g\rangle_{1}$, is prepared in state

$$
|\Psi\rangle_{1}=\sqrt{\frac{2}{3}}|g\rangle_{1}+\sqrt{\frac{1}{3}}|e\rangle_{1},
$$

by a classical pulse resonant with the $|g\rangle \rightarrow|e\rangle$ transition. The coefficients in the equation above are set adjusting the duration of the classical pulse to a $\phi=\arcsin (\sqrt{1 / 3})$ rotation (see Fig. 1). The production of (2) can be checked in auxilliary experiments measuring the population of states $|g\rangle_{1}$ and $|e\rangle_{1}$ and the quantum coherence. The atomic state (2) is then transferred to $C_{a}$, through a $\pi$ pulse of resonant quantum Rabi oscillation [18]. Atom $A_{1}$ finally leaves $C_{a}$ in state $|g\rangle_{1}$ and the cavity field is left in state $|\psi\rangle_{a}=\sqrt{2 / 3}|0\rangle_{a}+\sqrt{1 / 3}|1\rangle_{a}$. The first atom's final state being factored out, it will no longer be considered here.

Atom $A_{2}$, carrying the state to be cloned, crosses then $C_{a}$. It is prepared in the arbitrary state

$$
|\Psi\rangle_{2}=\alpha|+\rangle_{2}+\beta|-\rangle_{2},
$$

where $\alpha$ and $\beta$ are complex coefficients. Note that the preparation of this state, which is not part of the quantum cloning process, is not represented in figure 1. This atom interacts with the cavity field, performing a $2 \pi$ quantum Rabi pulse, amounting to a resonant quantum phase gate (QPG) described in [19]. The QPG produces a $\pi$ phase shift of the atom-cavity quantum state if and only if the atom is in state $|g\rangle$ and the cavity in state $|1\rangle_{a}$. When expressed in the $\{|+\rangle,|-\rangle\}$ basis, this QPG operation amounts to a controlled not gate (CNOT), where the control qubit is the field state. After this interaction the total entangled atom-field state becomes

$$
\sqrt{\frac{2}{3}}\left(\alpha|+\rangle_{2}+\beta|-\rangle_{2}\right)|0\rangle_{a}+\sqrt{\frac{1}{3}}\left(\alpha|-\rangle_{2}+\beta|+\rangle_{2}\right)|1\rangle_{a} .
$$

We then send a third atom, $A_{3}$, prepared in state $|g\rangle_{3}$. It interacts resonantly with $C_{a}$, for a time interval corresponding to a $\pi / 2$ quantum Rabi pulse, producing the state

$$
\begin{aligned}
& \sqrt{\frac{2}{3}}\left(\alpha|+\rangle_{2}+\beta|-\rangle_{2}\right)|g\rangle_{3}|0\rangle_{a} \\
& +\sqrt{\frac{1}{6}}\left(\alpha|-\rangle_{2}+\beta|+\rangle_{2}\right)\left(|g\rangle_{3}|1\rangle_{a}+|e\rangle_{3}|0\rangle_{a}\right) .
\end{aligned}
$$

The state of $C_{a}$ is finally transferred to a fourth atom, $A_{4}$, initially in $|g\rangle_{4}$ via a resonant $\pi$ quantum Rabi pulse, creating the three-atom entangled state:

$$
\begin{aligned}
& \sqrt{\frac{2}{3}}\left(\alpha|+\rangle_{2}+\beta|-\rangle_{2}\right)|g\rangle_{3}|g\rangle_{4} \\
& +\sqrt{\frac{1}{6}}\left(\alpha|-\rangle_{2}+\beta|+\rangle_{2}\right)\left(|g\rangle_{3}|e\rangle_{4}+|e\rangle_{3}|g\rangle_{4}\right),
\end{aligned}
$$

and leaving $C_{a}$ in the vacuum state, which factors out.

Classical microwave pulses then address the three atoms, transforming $|e\rangle$ into $|i\rangle$ via a two-photon $\pi$ pulse. This transformation does not affect state $|g\rangle$. Then, another classical $\pi / 2$ pulse is applied on the three atoms, combining states $|g\rangle$ and $|i\rangle$. The sequence of transformations produced by these classical pulses can be summarized as follows:

$$
\begin{aligned}
& |g\rangle \longleftrightarrow|g\rangle \longleftrightarrow \sqrt{\frac{1}{2}}(|i\rangle-|g\rangle)=|-\rangle, \\
& |e\rangle \longleftrightarrow|i\rangle \longleftrightarrow \sqrt{\frac{1}{2}}(|i\rangle+|g\rangle)=|+\rangle .
\end{aligned}
$$

The remaining part of the protocol involves the second cavity $C_{b}$. Atom $A_{2}$ interacts resonantly with $C_{b}$ for a time interval corresponding to a $\pi$ quantum Rabi pulse, transferring its state to the field mode. The final state of $A_{2}$ is $|g\rangle_{2}$ and also factorizes out. The total state of $A_{3}$, $A_{4}$ and $C_{b}$ is then :

$$
\begin{aligned}
& \sqrt{\frac{2}{3}}\left(\alpha|1\rangle_{b}+\beta|0\rangle_{b}\right)|-\rangle_{3}|-\rangle_{4} \\
& +\sqrt{\frac{1}{6}}\left(\alpha|0\rangle_{b}+\beta|1\rangle_{b}\right)\left(|-\rangle_{3}|+\rangle_{4}+|+\rangle_{3}|-\rangle_{4}\right) .
\end{aligned}
$$

Atoms $A_{3}$ and $A_{4}$ interact then independently and succesively with $C_{b}$. They perform a resonant QPG, corresponding to a CNOT in the $\{|+\rangle,|-\rangle\}$ basis. The final 
state of these three systems writes, after a simple rearrangement of terms:

$$
\begin{array}{r}
\alpha\left[\sqrt{\frac{2}{3}}|+\rangle_{3}|+\rangle_{4}|\mathcal{A}\rangle+\sqrt{\frac{1}{3}}|\Phi\rangle_{3,4}\left|\mathcal{A}_{\perp}\right\rangle\right] \\
+\beta\left[\sqrt{\frac{2}{3}}|-\rangle_{3}|-\rangle_{4}\left|\mathcal{A}_{\perp}\right\rangle+\sqrt{\frac{1}{3}}|\Phi\rangle_{3,4}|\mathcal{A}\rangle\right],
\end{array}
$$

where $|\mathcal{A}\rangle=|g\rangle_{1}|g\rangle_{2}|0\rangle_{a}|0\rangle_{b},\left|\mathcal{A}_{\perp}\right\rangle=|g\rangle_{1}|g\rangle_{2}|0\rangle_{a}|1\rangle_{b}$ and $|\Phi\rangle_{3,4}=\left(|+\rangle_{3}|-\rangle_{4}+|-\rangle_{3}|+\rangle_{4}\right) / \sqrt{2}$. In Eq. (9), the second cavity field ensures the orthogonality of $|\mathcal{A}\rangle$ and $\left|\mathcal{A}_{\perp}\right\rangle$ and hence is the important qubit in the ancilla's final state. This achieves the implementation of the optimal $1 \rightarrow 2$ cloning process.

Eq. (9) shows that this sequence actually implements the UCQM transformation given by Eq. (11). In this proposal, the blank state $|\mathcal{B}\rangle$ corresponds to the initial state of atoms $A_{1}, A_{3}$ and $A_{4}$ and of both cavity fields. It writes thus

$$
|\mathcal{B}\rangle=\left(\sqrt{\frac{1}{3}}|e\rangle_{1}+\sqrt{\frac{2}{3}}|g\rangle_{1}\right)|g\rangle_{3}|g\rangle_{4}|0\rangle_{a}|0\rangle_{b} .
$$

c. Discussion: We can now discuss the feasibility of an experimental implementation of the UQCM in a CQED system. The basic operations (quantum gates and classical field pulses) involved in the scheme have already been troughfully tested [16]. Their implementation thus does not present any major difficulty. The availability of an experimental configuration with two cavities can be considered as natural development of the present configurations using a single cavity, and mainly a matter of time. Note also some other interesting proposals require at least a two-cavity system 20].

Atoms interact with $C_{a}$ or $C_{b}$ for a time corresponding at most to a $2 \pi$ quantum Rabi pulse. The single photon Rabi frequency 18 being $\Omega / 2 \pi=50 \mathrm{kHz}$, the atomic velocity should be $\approx 500 \mathrm{~m} / \mathrm{s}$, in the range used in present experiments. Cavity and atomic relaxation are of course important issues. The circular Rydberg atoms lifetime is much longer than the protocol duration and is not bound to be a limiting factor. The main cause of decoherence in the present set-up is the cavity mode relaxation. The quantum information is stored in $C_{a}$ only during the time interval between the passage of $A_{1}$ and $A_{4}$. Each atom may enter the cavity immediately after the preceding one has left it. The total quantum information storage time is of the order of 4 full atomic transit times, i.e. $\approx 2 \cdot 10^{-4} \mathrm{~s}$. This is shorter than present cavity damping times (about $1 \mathrm{~ms}$ ). The cavity $C_{b}$ stores quantum information for an even shorter time interval. Note finally that the atomic transit time between the two cavities does not matter to evaluate the influence of damping, since the quantum information is then carried by long-lived atomic systems.

An alternative implementation of our UCQM scheme uses two modes of a single cavity. In the present experimental set-up, the cavity sustains two gaussian modes,
$M_{a}$ and $M_{b}$, with orthogonal linear polarizations. Due to mirrors imperfections, these two modes have slightly different resonant frequencies (splitting $130 \mathrm{kHz}$ ). Since this splitting is much larger than the atom-field coupling $\Omega$, the atoms resonantly interact with one mode only at at same time. Stark tuning can be used to tailor atomic interactions with the two modes during the atomic transit time through the cavity.

In this scheme, $A_{1}$ leaves its state in $M_{a}$. Then, $A_{2}$ performs the CNOT operation in $M_{a}$. It is set off-resonance with both modes for a short time interval during which the microwave classical pulses are applied. Atom $A_{2}$ it then tuned to resonance with $M_{b}$ for its final quantum Rabi pulse. $A_{3}$ and $A_{4}$ interact first resonantly with $M_{a}$, undergo the classical pulses while being off-resonance from the two modes and finally interact with $M_{b}$ as described above. This implementation of the UCQM, requiring a single cavity, would be much simpler to realize. Each atom should interact with the cavity for a total time corresponding at most to a $3 \pi$ quantum Rabi pulse (the duration of the classical pulses is negligible). The atomic velocity should be about $330 \mathrm{~m} / \mathrm{s}$, still well within the available range. The quantum information is stored in the cavity modes for a slightly longer time than in the two-cavity arrangement (four times the full transit time of atoms at the slower $330 \mathrm{~m} / \mathrm{s}$ velocity). Cavity damping should thus be somewhat smaller.

The UQCM operation verification can, in principle, be performed by the usual detection techniques [16]. As mentioned above, the fidelity is, ideally, $5 / 6$ while the trivial production of a maximally mixed state gives an average fidelity of $2 / 3$. This means that the fidelity should be measured with a precision greater than $\approx 92 \% \quad(=$ $\left.1-\frac{1}{2}(5 / 6-2 / 3)\right)$. Note that, in the NMR quantum cloning experiment [15], this degree of precision was not reached, so that the improvement due to the cloning process could not be verified.

In our proposal, all the elementary operations, quantum Rabi or classical field pulses, are prone to errors. The total number of these operations is sixteen if we take into account the detection and preparation process. The necessary precision could only be reached if each pulse has a fidelity greater than $\sqrt[16]{0.92}$. This value,being about 0.995 is still out of the experimental reach (present pulse imperfections are between 3 and 10\%). This figure, however, sets an interesting goal to be reached.

d. Conclusion. We described a protocol implementing the universal optimal copying transformation in CQED. Basic quantum information operations have already been implemented in this context [19], and proposals that could extend these experimental realizations to more elaborated quantum information algorithms 21] are naturally appealing.

The quantum logics network used in our scheme is simpler than previous ones by making use of auxiliary degrees of freedom which are discarded in the end of the process. Note also that the same protocol can be applied to the cloning of equatorial qubits [3], 
i.e., $|\psi\rangle=\sqrt{1 / 2}\left(|0\rangle+e^{i \phi}|1\rangle\right)$ by sending $A_{1}$ in state $\sqrt{1 / 2}(|e\rangle+|g\rangle)$.

The authors wish to thank J. Kempe for calling our attention to the problem and M. Brune and S. Haroche for inspiring discussions.

Laboratoire Kastler Brossel, Université Pierre et Marie Curie and ENS, is associated with CNRS (UMR 8552).

[1] W. K. Wooters and W. H. Zurek, Nature (London) 299, 802(1982); D. Dieks, Phys. Lett. 92A, 271 (1982).

[2] V. Buzek and M. Hillery, Phys. Rev. A 54, 1844 (1996).

[3] D. Bruss, et al., Phys. Rev. A 57, 2368 (1998); D. Bruss et al. Phys. Rev. A 62, 012302 (2000).

[4] N. J. Cerf, T. Durt, and N. Gisin, quant-ph/0110092 (2001).

[5] J. Fiurasek, S. Ibsidir, S. Massar, and N. J. Cerf, quantph/0110016 (2001).

[6] R. Josza, J. Mod. Optics 41, 2315 (1994).

[7] N. Gisin and S. Massar, Phys. Rev. Lett. 79, 2153 (1997).

[8] K. Banaszek, Phys. Rev. Lett. 86, 1366 (2001); D. Bruss, A. Ekert and C. Macchiavello, Phys. Rev. Lett. 81 , 2598 (1998).

[9] F. Grosshans and Ph. Grangier, Phys. Rev. A 64, 010301(R) (2001).

[10] E. F. Galvão and L. Hardy, Phys. Rev. A 62, 022301 (2000).

[11] C. Simon, G. Weihs, and A. Zeilinger, Phys. Rev. Lett. 84, 2993 (2000); J. Kempe, C. Simon, and G. Weihs, Phys. Rev. A 62, 032302 (2000).

[12] V. Buzek, S. L. Braunstein, M. Hillery, and D. Bruss, Phys. Rev. A 56, 3446 (1997).

[13] Y.-F. Huang et al., Phys. Rev. A 64, 012315 (2001).

[14] A. Lamas-Linares, C. Simon, J. Howell, and D. Bouwmeester, Science 286, 712 (2002);S. Fasel et al, quant-ph/0203056 (2002).

[15] H. K. Cummins et al., Phys. Rev. Letters 88, 187901 (2002).

[16] J. M. Raimond, M. Brune, and S. Haroche, Rev. Mod. Phys. 73, 565 (2001).

[17] A. Rauschenbeutel, P. Bertet, S. Osnaghi, G. Nogues, M. Brune, J. M. Raimond, and S. Haroche, Phys. Rev. A 64, 050301(R) (2001); P. Bertet, S. Osnaghi, P. Milman, A. Auffeves, P. Maioli, M. Brune, J. M. Raimond, and S.
Haroche, Phys. Rev. Lett. 88, 143601 (2002).

[18] X. Maitre et al., Phys. Rev. Lett. 79, 769 (1997); M. Brune et al., Phys. Rev. Lett. 76, 1800 (1995).

[19] A. Rauschenbeutel et al., Phys. Rev. Lett. 83, 5166 (1999).

[20] L. Davidovich, N. Zagury, M. Brune, J. M. Raimond and S. Haroche, Phys. Rev. A 50, R895 (1994).

[21] F. Yamaguchi, P. Milman, M. Brune, J. M. Raimond, and S. Haroche, to be published in Phys. Rev. A; M. O. Scully and M. S. Zubairy, Phys. Rev. A 65, 052324 (2002).

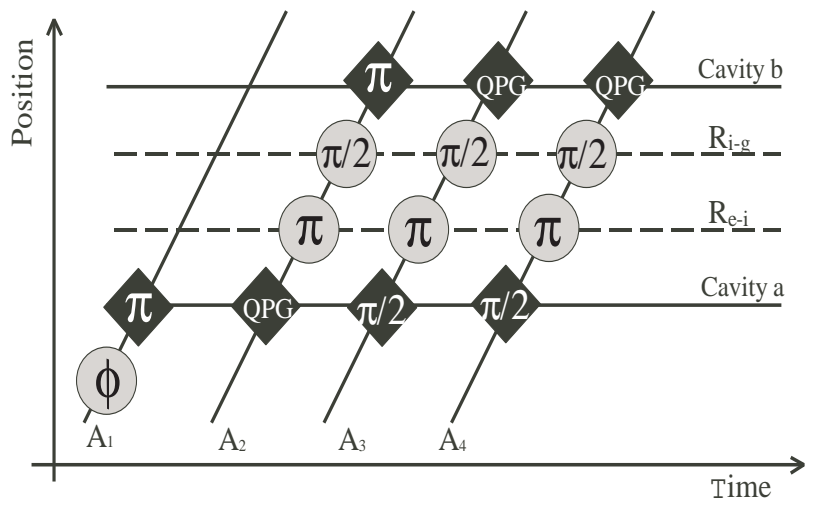

FIG. 1. Detailed scheme of the atom field interactions in each cavity. $A_{1}$ enters the first cavity in state $\sqrt{1 / 3}|e\rangle_{1}+\sqrt{2 / 3}|g\rangle_{1}$ via a classical pulse of a certain duration and transfers its state to the cavity field in a $\pi$ Rabi pulse. The cavity performs thus a QPG in $A_{2}$, the atom carrying the state to be cloned $\alpha|+\rangle_{2}+\beta|-\rangle_{2}$. After being manipulated by microwave classical fields, $A_{2}$ also transfers its state to the second cavity, which is now entangled to the first one. A third atom $A_{3}$, prepared in state $|g\rangle_{3}$, crosses the first cavity performing a $\pi / 2$ Rabi pulse. The first cavity field's state is completely recovered by the atomic states via the passage of a fourth atom, $A_{4}$, also prepared in $|g\rangle_{4}$, which makes a $\pi$ Rabi pulse. Both of these atoms will interact resonantly with the second cavity field after classical microwave pulses manipulation. They will perform a resonant QPG, which will leave the total combined atom+second cavity field in the desired final state, corresponding to the cloning transformation. 